\title{
Uma Única Observação da Pressão Plantar Representa a Marcha de Crianças em Diferentes Dias?
}

\section{Does a Single Plantar Pressure Observation Represent Children's gait in Different Days?}

\author{
Mariane de Medeiros Figueiredo ${ }^{1}$ \\ Pedro Silvelo Franco ${ }^{2}$ \\ Cristiane Fraga Moro $^{3}$ \\ Emmanuel Souza da Rocha ${ }^{4}$ \\ Álvaro Sosa Machado ${ }^{5}$ \\ Felipe Pivetta Carpes $^{6}$
}

\section{RESUMO}

Objetivo: Determinar a variabilidade, a repetibilidade e as assimetrias da pressão plantar na marcha de crianças avaliadas em diferentes dias. Metodologia: Participaram deste estudo 12 crianças voluntárias ( 6 meninos) com idade média de 10,0 $\pm 2,0$ anos. Elas caminharam descalças em velocidade preferida ao longo de um trajeto de $9 \mathrm{~m}$. A avaliação da marcha foi repetida em 4 dias não consecutivos de uma semana e a pressão plantar foi monitorada em cada um dos dias através de um baropodômetro. Os dados de pressão plantar foram comparados entre as pisadas em cada dia e entre os diferentes dias, considerando a pressão média e o pico de pressão para cada um dos pés. $O$ índice de assimetria foi quantificado e comparado entre os dias. Resultados: As crianças tiveram um padrão consistente para pressão média e pico de pressão plantar. Os índices de assimetria observados foram similares entre os diferentes dias avaliados. Conclusão: A pressão média e o pico de pressão apresentaram valores consistentes em diferentes dias em que crianças são avaliadas, o que inclui similar assimetrias nos diferentes dias.

\section{DESCRITORES}

Cinética. Infância. Biomecânica. Pé. Caminhada.

\begin{abstract}
Objective: To determine the variability, repeatability, and symmetry of plantar pressure in the children gait along different days. Methodology: Twelve children ( 6 boys) participated in this study. They walked barefoot at preferred gait speed along $9 \mathrm{~m}$. Gait was assessed in 4 non-consecutive days within a period of 7 days, and the plantar pressure was monitored using a pressure mat. Plantar pressure data were compared between the steps and different days considering mean and peak pressures for each foot. The asymmetry index was also quantified and compared between the days. Results: Children in our study showed a consistent pattern for mean and peak pressure. Asymmetry indexes were similar in the different days. Conclusion: Mean and peak pressure showed to be consistent when children are assessed in different days, which includes similar asymmetries across the different days.
\end{abstract}

\section{DESCRIPTORS}

Kinetic. Infants. Biomechanics. Foot. Walk.

\footnotetext{
${ }^{1}$ Fisioterapeuta, Grupo de Pesquisa em Neuromecânica Aplicada, Laboratório de Neuromecânica, Universidade Federal do Pampa, Uruguaiana, RS, Brasil.

${ }^{2}$ Mestre em Educação Física, Grupo de Pesquisa em Neuromecânica Aplicada, Laboratório de Neuromecânica, Universidade Federal do Pampa, Uruguaiana, RS, Brasil.

${ }^{3}$ Pesquisadora Associada, Grupo de Pesquisa em Neuromecânica Aplicada, Laboratório de Neuromecânica, Universidade Federal do Pampa, Uruguaiana, RS, Brasil.

${ }^{4}$ Mestre em Educação Física, Doutorando em Ciências do Movimento Humano, Grupo de Pesquisa em Biomecânica e Cinesiologia, Laboratório de Pesquisa do Exercício, Universidade Federal do Rio Grande do Sul, Porto Alegre, RS, Brasil.

${ }^{5}$ Fisioterapeuta, Mestrando em Bioquímica, Grupo de Pesquisa em Neuromecânica Aplicada, Laboratório de Neuromecânica, Universidade Federal do Pampa, Uruguaiana, RS, Brasil.

${ }^{6}$ Doutor em Ciências do Movimento Humano, Professor Adjunto, Grupo de Pesquisa em Neuromecânica Aplicada, Laboratório de Neuromecânica, Universidade Federal do Pampa, Uruguaiana, RS, Brasil.
} 
$\mathrm{N}$ o nascimento, a estrutura mecânica do pé humano baseia-se predominantemente em tecidos moles. A partir do momento em que o pé da criança passa a experimentar cargas mecânicas resultantes da busca por uma postura em pé, e devido ao aumento da massa corporal, ocorrem adaptações morfológicas e funcionais nos pés, que vão atuar no suporte e na dissipação de cargas durante a marcha ${ }^{1,2}$. Apesar de meninos apresentarem pés maiores, o padrão de desenvolvimento é equivalente para ambos os sexos ${ }^{3}$.

Essa interação do pé com a superfície vem sendo analisada extensivamente na prática clínica com o emprego dos baropodômetros, que são equipamentos úteis para a avaliação da pressão plantar durante a marcha ${ }^{4}$. Na prática clínica, essas avaliações muitas vezes buscam detectar deformidades anatômicas e o risco de lesões plantares ${ }^{4,5}$. Dentre as variáveis frequentemente utilizadas para a quantificação da pressão plantar em crianças estão as medidas de pressão média e o pico de pressão durante a marcha ${ }^{6}$.

Essas medidas geralmente são realizadas em um único dia de avaliação, a partir da tomada de um determinado número de pisadas. Contudo, existem fatores que podem comprometer essa medida transversal sendo tomada como representativa do padrão de pressão plantar para aquela criança avaliada. Por exemplo, a familiarização com a avaliação e a compreensão das explicações do avaliador, assim como a motivação e a atenção ${ }^{7}$ podem influenciar as medidas $^{8}$. Será então que essa medida de um dia consegue representar um padrão de diferentes dias?
Logo, será que é necessário realizar mais de uma avaliação da marcha para determinar o padrão da pressão plantar de crianças?

Em idosos estas perguntas vêm sendo consideradas. Em um estudo prévio, a variabilidade, a repetibilidade e as assimetrias na pressão plantar média e no pico de pressão durante a marcha de idosos se mostraram consistentes em diferentes dias de avaliação, mantendo também consistentes as assimetrias observadas ${ }^{9}$. Contudo, a generalização dos resultados encontrados, para a avaliação de crianças, pode ser limitada devido as diferenças nos padrões de caminhada ${ }^{10,11}$.

Adicionalmente, a repetibilidade dos padrões de pressão também influencia a simetria da marcha, uma vez que a repetibilidade de assimetrias, mesmo que de baixa magnitude, pode representar sobrecargas experimentadas, repetidamente, em longo prazo e que podem resultar em lesões por estresse $^{12}$.

Portanto, sabendo da escassez de trabalhos que investiguem a repetibilidade de padrões de pressão plantar em crianças e da necessidade de avaliações biomecânicas apresentarem dados consistentes para uso no diagnóstico de alterações nos pés de crianças, o objetivo deste estudo foi avaliar a repetibilidade da pressão plantar, incluindo assimetrias, durante a marcha de crianças em diferentes dias. Com isso buscamos, também, verificar se a avaliação da pressão plantar realizada em crianças, em uma única sessão de avaliação da marcha, é suficiente para representar o seu padrão de pressão plantar.

$$
I A(\%)=\left(\frac{P-N P}{P}\right) \times 100
$$




\section{METODOLOGIA}

Participaram deste estudo 12 crianças voluntárias, sendo 6 meninos e 6 meninas (Tabela 1) da comunidade local e que apresentaram o Termo de assentimento e de consentimento livre e esclarecido, assinado pelo responsável. Todas as crianças incluídas no estudo foram capazes de participar das quatro avaliações em um período de no máximo 7 dias, sendo as avaliações realizadas em dias não consecutivos. Para participar, as crianças deveriam ser capazes de deambular sem qualquer auxílio. Os critérios de exclusão envolveram lesão de qualquer um dos membros inferiores no último ano, bem como presença de doenças neuromusculares que afetassem a marcha ${ }^{4}$. A pesquisa foi conduzida dentro dos padrões exigidos pela Declaração de Helsinque e da Resolução e da Resolução no. 466/12, do Conselho Nacional de Saúde (CNS), que estabelece as diretrizes e as normas reguladoras envolvendo seres humanos. Este estudo foi aprovado pelo comitê de ética da universidade local (Protocolo $n^{\circ}$. 062011).

\section{Delineamento Experimental}

As crianças foram avaliadas durante o andar descalço, em linha reta e em veloci- dade preferida, ao longo de um trajeto de 9 $\mathrm{m}$. As informações da pressão plantar foram registradas por meio de um tapete sensorizado, posicionado na metade do trajeto onde a caminhada era realizada. A velocidade foi determinada pelo tempo gasto pelo participante para percorrer a distância de $9 \mathrm{~m}$. O protocolo da marcha foi repetido em quatro dias não consecutivos, em um intervalo de no máximo 7 dias. Para cada dia de avaliação, a pressão plantar foi gravada para 10 pisadas com cada pé. Buscou-se realizar a avaliação da marcha sempre na mesma hora e turno do dia. Os dados foram comparados entre as pisadas em cada dia e entre os diferentes dias de avaliação.

\section{Avaliação da pressão plantar}

Um baropodômetro computadorizado em formato de tapete (Matscan, Tekscan Inc., Boston, EUA, com resolução de 1,4 sensores por $\mathrm{cm}^{2}$, totalizando 2.288 sensores) foi utilizado para o registro da pressão plantar. Os dados foram gravados com uma taxa de amostragem de $400 \mathrm{~Hz}$. As medidas da pressão plantar foram realizadas para determinar a pressão média e o pico de pressão, considerando a totalidade de área de contato do pé com o solo. $O$ índice de assimetria foi calculado pela equação $1^{13}$.

Tabela 1. Caracterização das crianças incluídas no estudo

\begin{tabular}{l|c}
\hline \multicolumn{1}{c|}{ Características $(\mathrm{n}=12)$} & Média \pm desvio-padrão \\
\hline Idade (anos) & $10 \pm 2$ \\
Massa corporal $(\mathrm{kg})$ & $50 \pm 18$ \\
Estatura $(\mathrm{m})$ & $1,48 \pm 0,16$ \\
$\mathrm{IMC}\left(\mathrm{kg} / \mathrm{m}^{2}\right)$ & $21 \pm 5$ \\
\hline
\end{tabular}


Equação 1, onde IA significa índice de assimetria; $\mathrm{P}$ representa o valor da perna preferida e NP o valor da perna não preferida.

O tapete instrumentado ficava disposto no meio do trajeto da caminhada, de modo a minimizar efeitos da aceleração e desaceleração durante a marcha. Os participantes eram orientados a caminhar sem se preocupar em pisar ou não no tapete. As pisadas em que o participante colocasse todo o pé no tapete, sem que o pé contralateral tocasse no aparelho, foram consideradas válidas. Pisadas com o pé direito e o esquerdo eram gravadas aleatoriamente, até que 10 pisadas com cada pé fossem gravadas. A partir das informações da pressão plantar, foi determinada a pressão média e o pico de pressão para a área total de cada pé, em cada pisada.

Para os dados da pressão plantar (média e pico), em cada dia de avaliação, as informações foram organizadas em média \pm desvio padrão, considerando os valores de 10 pisadas em cada dia e em cada pé. Os dados foram comparados entre as 10 pisadas em cada um dos dias de avaliação e a média das 10 pisadas de cada dia foram comparadas entre os diferentes dias de avaliação. $O$ coeficiente de variação (CV) foi determinado considerando a razão entre o desvio padrão e a média. Para verificar a normalidade da distribuição dos dados usamos o teste de Shapiro-Wilk. Anova one-way com post-hoc de Tukey foi empregada para comparar as 10 pisadas em cada dia e entre os quatro dias de avaliação, para o pé direito e o esquerdo. Para expressar a magnitude das assimetrias em cada dia, calculamos o índice de assimetria ${ }^{12}$. O nível de significância foi estabelecido em 0,05 . As análises estatísticas foram realizadas utilizando o pacote estatístico SPSS versão 17.0.

\section{RESULTADOS}

A velocidade preferida dos participantes foi de 1,21 $\pm 0,09 \mathrm{~m} / \mathrm{s}$ e não diferiu entre os diferentes dias de avaliação.

\section{Comparação entre diferentes pisadas}

Quando a pressão média (Figura 1) foi comparada entre as 10 pisadas em cada dia de avaliação, não observamos diferenças entre as pisadas do pé direito $\left(F_{(9)}=0,4359\right.$; $P=0,952 ; F_{(9)}=1,091 ; P=0,376 ; F_{(9)}=0,498$; $P=0,873 ; F_{(9)}=0,814 ; P=0,604$ para os dias $1,2,3$ e 4, respectivamente) e pé esquerdo $\left(F_{(9)}=0,529 ; P=0,851 ; F_{(9)}=0,528 ; P=0,852\right.$; $F_{(9)}=0,702 ; P=0,706 ; F_{(9)}=0,341 ; P=0,959$; para os dias 1, 2, 3 e 4, respectivamente).

Quando a mesma comparação foi realizada levando em consideração o pico de pressão (Figura 2), também não observamos diferenças entre as pisadas com o pé direito $\left(F_{(9)}=0,745 ; P=0,667 ; F_{(9)}=0,463 ; P=0,897\right.$; $F_{(9)}=0,316 ; P=0,968 ; F_{(9)}=0,570 ; P=0,819$; para os dias 1, 2, 3 e 4, respectivamente) ou com o pé esquerdo $\left(F_{(9)}=0,360 ; P=0,952\right.$; $F_{(9)}=0,153 ; P=0,998 ; F_{(9)}=0,755 ; P=0,658$; $F_{(9)}=0,281 ; P=0,979 ;$ para os dias 1, 2, $3 \mathrm{e}$ 4 , respectivamente).

\section{Comparação entre diferentes dias}

Quando os dados da pressão plantar para cada pé, considerando então a média de 10 pisadas em cada dia (Figura 3), foram comparados entre os quatro diferentes dias de 
Figura 1. Média (barras) e desvio-padrão (linhas verticais) para a pressão média $\left(\mathrm{N} / \mathrm{cm}^{2}\right)$ considerando as 10 pisadas em cada dia, para a perna direita (superior) e esquerda (inferior)

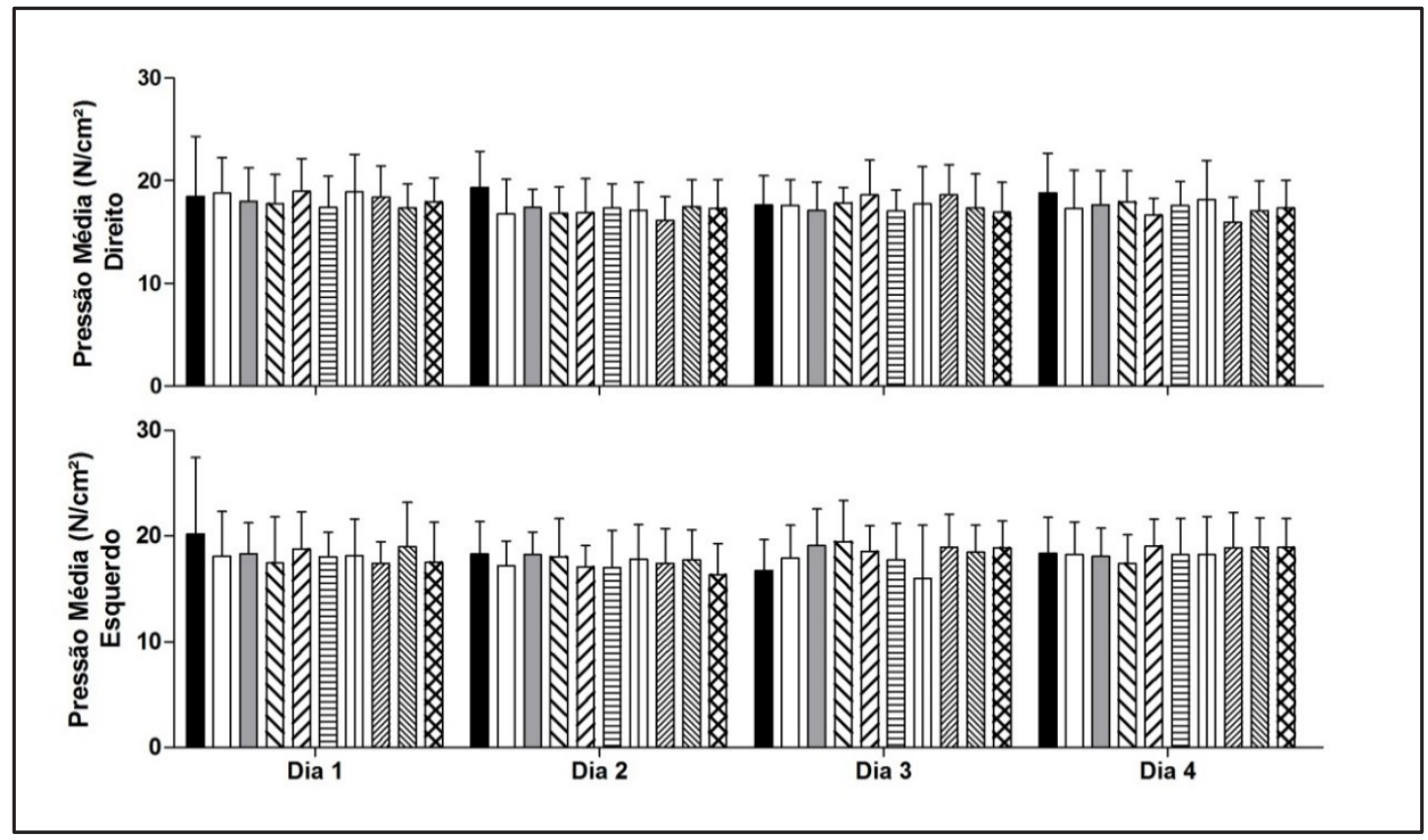

Figura 2. Média (barras) e desvio-padrão (linhas verticais) para o pico de pressão $\left(\mathrm{N} / \mathrm{cm}^{2}\right)$ considerando as 10 pisadas em cada dia, para a perna direita (superior) e esquerda (inferior)

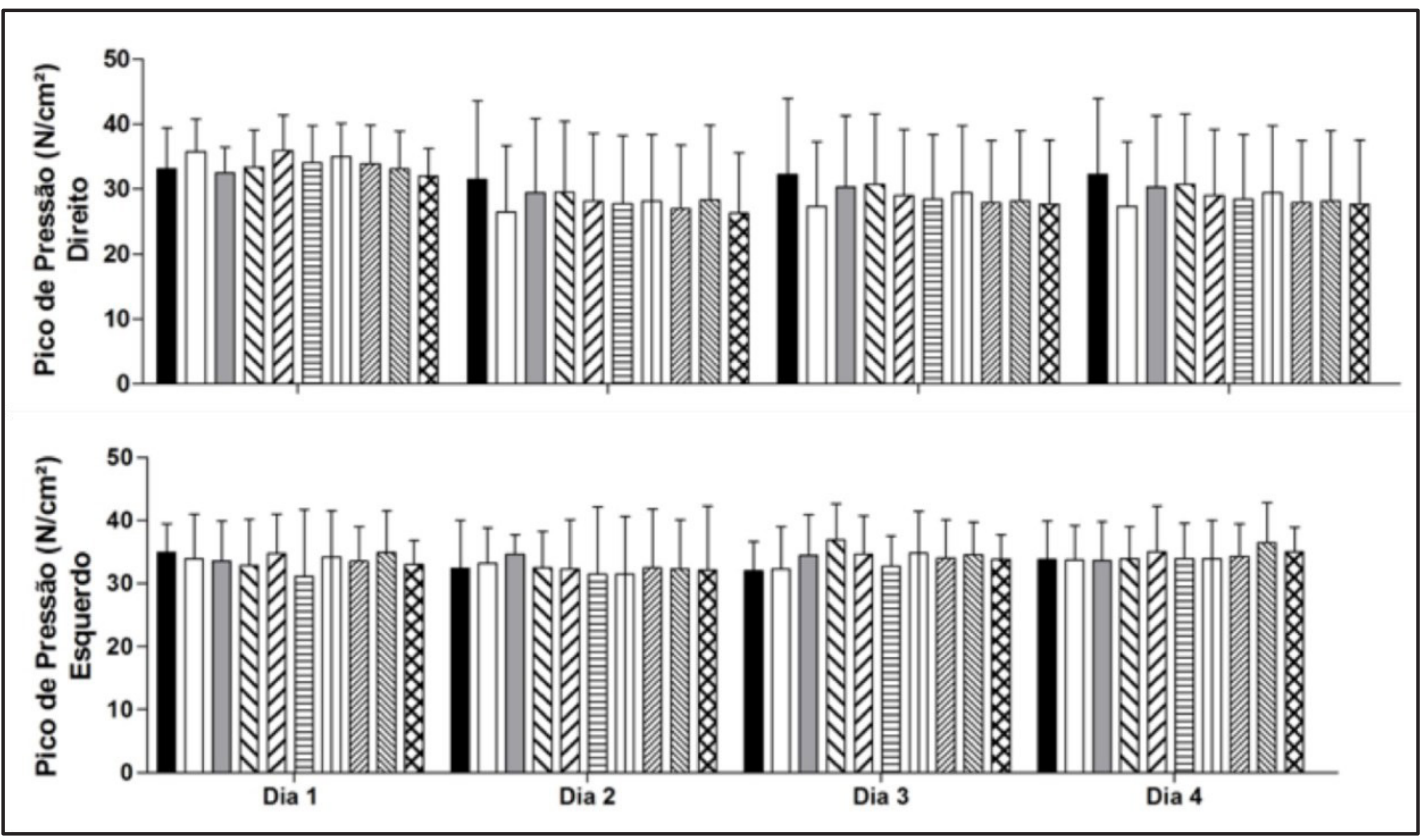


avaliação, observamos um padrão consistente na aplicação da pressão média, tanto para $O$ pé direito $\left(F_{(3)}=0,597 ; P=0,620\right)$ quanto para o pé esquerdo $\left(F_{(3)}=0,947 ; P=0,426\right)$. Similar resultado foi observado para o pico de pressão, tanto para o pé direito $\left(F_{(3)}=0,202 ; \mathrm{P}\right.$ $=0,894)$ quanto para o esquerdo $\left(F_{(3)}=0,316\right.$; $P=0,813)$.
Quando à variabilidade da pressão plantar (Figura 3) foi comparada entre os diferentes dias, observou-se um padrão consistente de variabilidade na pressão média ao longo dos quatro dias, tanto para o pé direito $\left(F_{(3)}=1,180 ; P=0,328\right)$ quanto para o pé esquerdo $\left(F_{(3)}=0,652 ; P=0,586\right)$. Este padrão também foi observado para a variabilidade do

Figura 3. Média (barras) e desvio-padrão (linhas verticais) para a pressão média $\left(\mathrm{N} / \mathrm{cm}^{2}\right)$, variabilidade da pressão média $(\mathrm{CV} \%)$, pico de pressão $\left(\mathrm{N} / \mathrm{cm}^{2}\right)$ e variabilidade do pico de pressão (CV\%), do topo para o fim da figura, respectivamente

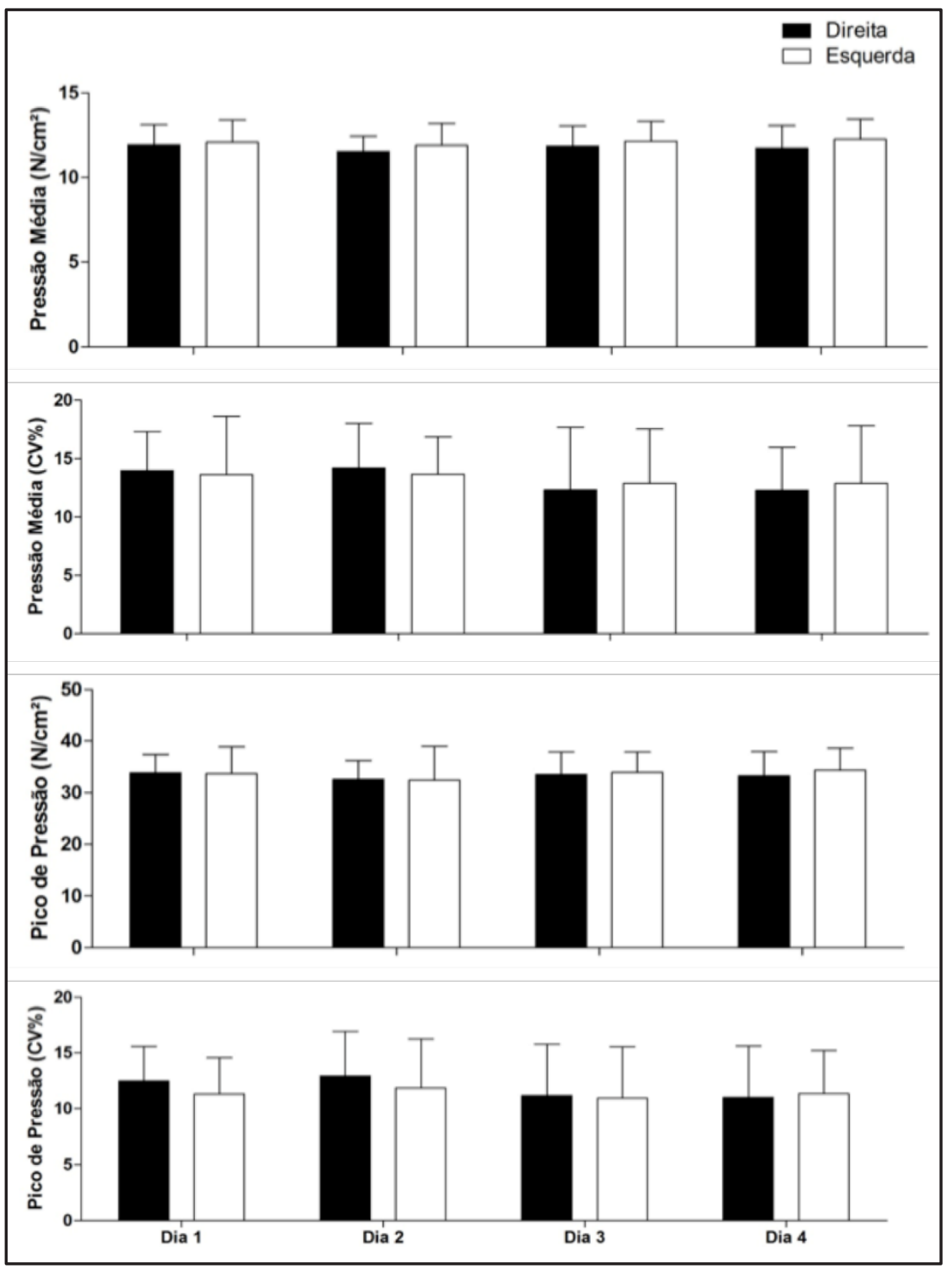


pico de pressão no pé direito $\left(F_{(3)}=0,640 ; P=\right.$ $0,593)$ e no esquerdo $\left(F_{(3)}=0,693 ; P=0,561\right)$. $O$ índice de assimetria observado para a pressão média foi de $7,53 \% \pm 4,68$ nos diferentes dias (Figura 4). Para o pico de pressão, o índice de assimetria foi de 6,31\% $\pm 4,85$. Tanto para a pressão média quanto para o pico, os índices de assimetria não diferiram entre os quatro dias de avaliação $\left(F_{(3)}\right.$ $=1,713 ; \mathrm{P}=0,178$ e $\mathrm{F}_{(3)}=0,129 ; \mathrm{P}=0,943$, para a pressão média e o pico de pressão, respectivamente). Ao compararmos o índice de assimetria observado para a pressão média e para o pico de pressão, não foram encontradas diferenças significativas para nenhum dia de avaliação (Figura 4).

\section{DISCUSSÃO}

A análise da pressão plantar é usada para prover informações sobre a distribuição de cargas mecânicas sobre os pés. Em virtude do seu uso em ambientes clínicos e de pesquisa, é preciso assegurar que protocolos para avaliação da pressão plantar sejam confiáveis para reproduzir as características da pressão plantar dinâmica em diferentes ocasiões. Neste estudo determinamos a variabilidade, a repetibilidade e a simetria da pressão plantar durante a marcha em crianças. Nosso principal achado foi que a pressão média e o pico de pressão, analisados em um dia, podem representar o padrão de pressão plantar de crianças. Adicionalmente, os índices de assimetria observados também se mostram similares em diferentes dias de avaliação.

Os resultados obtidos indicam que a pressão média e o pico de pressão, determinados considerando a totalidade da área de contato dos pés com o solo, são bastante consistentes entre os diferentes dias de avaliação. Adicionalmente, esse padrão consistente observado para ambos os pés acabou refletindo índices de assimetrias com poucas alterações ao longo de diferentes dias de avaliação. Os desvios de simetria observados ainda requerem investigação futura, para uma melhor compreensão dos seus impactos sobre os pés de crianças.

Figura 4. Média (barras) e desvio-padrão (linhas verticais) para o índice de assimetria (\%) nos quatro dias de avaliação para a pressão média (barras pretas) e pico de pressão (barras cinzas)

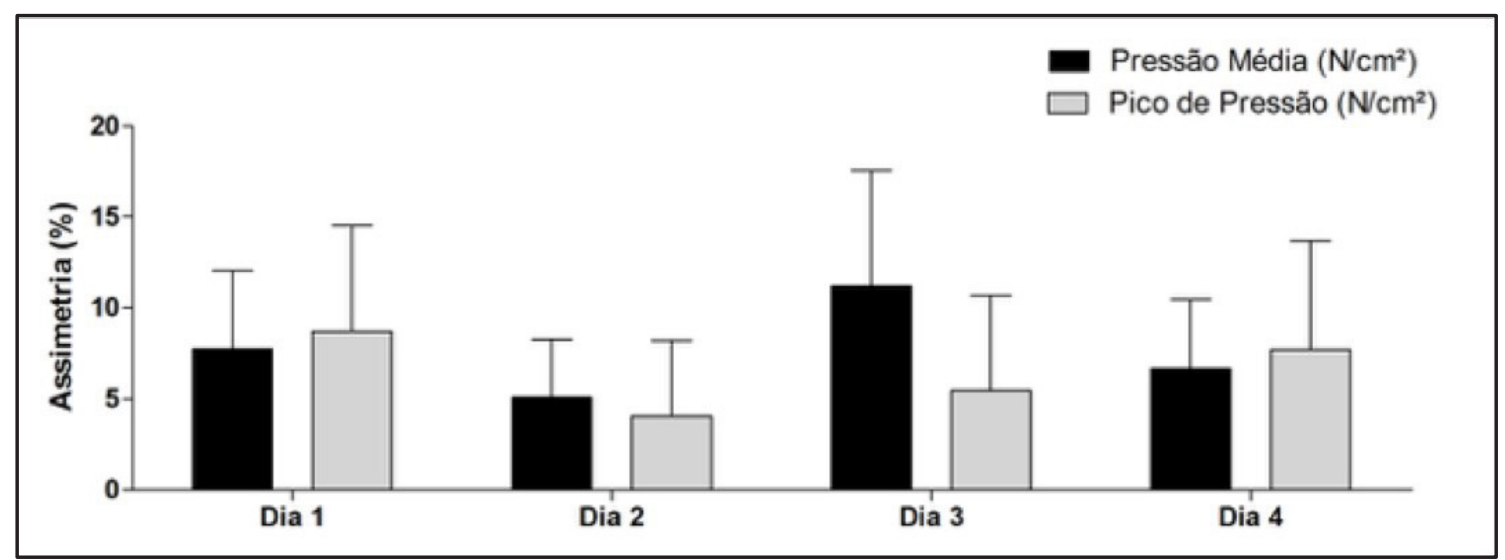


Quando analisamos as pisadas em um mesmo dia, encontramos um padrão consistente de média e de pico de pressão. Deepashini et al. ${ }^{14}$ avaliaram a confiabilidade da avaliação da pressão plantar com baropodômetros e constataram que o pico de pressão pode ser o parâmetro mais adequado para sugerir uma boa confiabilidade na avaliação da pressão plantar entre dias distintos. Em nosso estudo, em adição ao pico, vimos que a média da pressão, considerando a área do pé inteiro, também pode apresentar um padrão reprodutível.

Pequenas variações na faixa etária das crianças parecem influenciar a repetibilidade da marcha, o que é percebido em estudos que realizaram diferentes medidas. A consistência dos resultados encontrados neste estudo coincide, em parte, com o estudo de Thorpe et al. ${ }^{7}$. Estes autores analisaram a repetibilidade dos parâmetros espaço-temporais da marcha de crianças saudáveis e encontraram níveis de repetibilidade satisfatórios para crianças de 4 a 8 anos. Porém, para crianças de 8 a 11 anos o nível de repetibilidade foi menor, o que pode ter resultado do fato de que as crianças nessa faixa etária sentiam-se mais à vontade no segundo dia de teste e, talvez, por isso, alteraram o padrão de marcha ${ }^{7}$. No estudo de Beck et al. ${ }^{15}$ a avaliação da marcha de crianças com idade acima de 5 anos também evidenciou um padrão satisfatório de repetibilidade na força de reação do solo. Além disso, após um período de três meses, não se observou variação nas medidas. Contudo, para crianças com menos de 4 anos de idade, as medidas apresentaram pouca repetibilidade ${ }^{15}$. Uma possível explicação para essas diferenças na repetibilidade entre as idades pode ser os diferentes níveis de desenvolvimento motor que as crianças se encontram e, também, no fato de que crianças com mais idade podem compreender melhor as orientações para a avaliação, o que ajuda na realização dos experimentos.

A velocidade da marcha pode ser uma fonte de variabilidade nos dados biomecânicos da extremidade inferior ${ }^{16}$. Em nosso estudo, optamos por avaliar as crianças em sua velocidade de marcha preferida, com o intuito de interferir o mínimo possível nos padrões. Observando a velocidade preferida das crianças que avaliamos, o coeficiente de variação foi de aproximadamente $7 \%$, o que revela uma baixa variabilidade. Em outro estudo, em que a velocidade de marcha foi pré-determinada ${ }^{17}$, observou-se que ocorreu um aumento no pico de pressão sobre o retropé e na parte medial do antepé e uma diminuição no mediopé e no antepé lateral, com o aumento da velocidade. Contudo, nosso estudo tem interesse na aplicabilidade na clínica e na simplificação da avaliação, o que torna mais interessante considerar a velocidade preferida, que, além do mais, se mostrou consistente ao longo dos dias.

Em nosso estudo não foi levado em consideração a existência de algum efeito do sexo sobre a análise dos dados que apresentamos, ainda que tenhamos buscado uma equivalência entre meninos e meninas em nosso grupo de estudo. Hennig et al. ${ }^{18}$ não encontraram diferença significativa entre meninos e meninas, com idade entre 6 e 10 anos, para a variável pico de pressão plantar. Por isso, consideramos que não existiria necessidade de estratificar os dados por sexo em nosso estudo. 
Nossos resultados revelaram um índice de assimetria de baixa magnitude, mas que se mostrou bastante consistente ao longo dos diferentes dias de avaliação. Conforme discutido em vários estudos, como por exemplo, o trabalho de Chavet et al. ${ }^{12}$, o impacto e a repetição de um padrão assimétrico de forças pode ser mais prejudicial do que sua própria magnitude. Isso pode ser especialmente importante se considerarmos que a pressão pode representar a atuação de cargas mecânicas sobre a estrutura anatômica do membro inferior. Pensando em analisar essa questão, Holm et al. ${ }^{19}$ encontraram padrões simétricos para variáveis como a pressão plantar média de crianças de 7 a 10 anos de idade. Neste estudo, foi descrito um índice de assimetria (IA) que foi similar ao longo dos dias e das pisadas. Embora o nosso objetivo não tenha sido investigar riscos de lesão associados com esses pequenos desvios de simetria, a repetibilidade das assimetrias pode ser prejudicial e representar um risco de lesão ${ }^{12}$. No estudo de Mickle et al. ${ }^{20}$ foi verificado um padrão similar ao do nosso estudo, onde os autores avaliaram assimetrias da marcha de crianças praticantes de atividade física e o quanto que esses desvios de simetria influenciam os riscos de lesão requer investigação adicional.

Putti et al. ${ }^{4}$ defendem a necessidade da realização de avaliações de pressão plantar em mais de um dia, pois consideram esta uma aproximação mais confiável quando comparada com apenas uma avaliação. Eles verificaram que três avaliações seriam suficientes para garantir representatividade do padrão da pressão plantar da marcha em crianças. Contudo, nossos dados sugerem que o padrão de pressão plantar em crianças pode ser satisfatoriamente descrito em um único dia de avaliação, considerando-se a pressão média e o pico de pressão na área total do pé.

A realização das medidas deste estudo se deu em vários dias e buscou-se um intervalo similar entre as avaliações, isso acabou limitando o número de participantes incluídos, pois várias crianças desistiram de participar das avaliações, após a tomada da primeira ou segunda medida. Foi muito difícil conseguir que as crianças completassem as quatro avaliações dentro dos requisitos de dias. A avaliação da pressão plantar descalço é uma limitação, mas fizemos essa escolha para que nossos dados tivessem minimizadas as influências dos diferentes tipos de calçados usados pelas crianças. Para o estudo, somente foram recrutadas crianças com desenvolvimento normal, o que indica que a as nossas conclusões não podem ser generalizadas para uma população atípica.

\section{CONCLUSÃO}

A avaliação da pressão plantar realizada em um dia parece ser suficiente para representar o padrão de pressão plantar na área total do pé em crianças, assim como assimetrias na locomoção em relação à pressão plantar.

\section{AGRADECIMENTOS}

Os autores agradecem aos participantes do estudo e a equipe do Grupo de Pesquisa em Neuromecânica Aplicada pelo auxílio nas fases experimentais. Durante o desenvolvimento deste estudo MMF e CFM receberam bolsa PROEXT-MEC. PSF recebeu bolsa de mestrado da CAPES. 


\section{REFERÊNCIAS}

1. Bertsch C, Unger H, Winkelmann W, Rosenbaum D. Evaluation of early walking patterns from plantar pressure distribution measurements. First year results of 42 children. Gait Posture. 2004; 119(3):235-242.

2. Bosch K, Rosenbaum D. Gait symmetry improves in childhood--a 4-year follow-up of foot loading data. Gait Posture. 2010; 132(4):464-468.

3. Gould N, Moreland M, Trevino S, Alvarez R, Fenwick J, Bach N. Foot growth in children age one to five years. Foot Ankle. 1990; 110(4):211-213.

4. Putti AB, Arnold GP, Cochrane LA, Abboud RJ. Normal pressure values and repeatability of the Emed ST4 system. Gait Posture. 2008; 127(3):501-505.

5. Kellis E. Plantar pressure distribution during barefoot standing, walking and landing in preschool boys. Gait Posture. 2001; 114(2):92-97.

6. Mickle KJ, Steele JR, Munro BJ. Does excess mass affect plantar pressure in young children? Int J Pediatr Obes. 2006; 11(3):183-188.

7. Thorpe DE, Dusing SC, Moore CG. Repeatability of temporospatial gait measures in children using the GAlTRite electronic walkway. Arch Phys Med Rehabil. 2005; 186(12):2342-2346.

8. Cousins SD, Morrison SC, Drechsler WI. The reliability of plantar pressure assessment during barefoot level walking in children aged 7-11 years. J Foot Ankle Res. 2012; 15(1):8

9. Franco PS, Silva CB, Rocha ES, Carpes FP. Variability and repeatability analysis of plantar pressure during gait in older people. Rev Bras Reumatol. 2015; 155(5):427-433.

10. Tong JW, Kong PW. Reliability of footprint geometric and plantar loading measurements in children using the Emed((R)) M system. Gait Posture. 2013; 138(2):281-286.

11. Akins JS, Keenan KA, Sell TC, Abt JP, Lephart SM. Test-retest reliability and descriptive statistics of geometric measurements based on plantar pressure measurements in a healthy population during gait. Gait Posture. 2012; 135(1):167-169.

12. Chavet $\mathrm{P}$, Lafortune MA, Gray JR. Asymmetry of lower extremity responses to external impact loading. Human Movement Science. 1997; 116(4):391-406.
13. Carpes FP, Mota CB, Faria IE. On the bilateral asymmetry during running and cycling - a review considering leg preference. Phys Ther Sport. 2010; 111(4):136-142.

14. Harithasan Deepashini BO, Aatit Paungmali, Naicker Amaramalar, Htwe Ohnmar, Joseph Leonard. An insight into the plantar pressure distribution of the foot in clinical practice: Narrative review. Polish Annals of Medicine. 2014; 121:51-56.

15. Beck RJ, Andriacchi TP, Kuo KN, Fermier RW, Galante JO. Changes in the gait patterns of growing children. J Bone Joint Surg Am. 1981; 163(9):1452-1457.

16. Keller TS, Weisberger AM, Ray JL, Hasan SS, Shiavi RG Spengler DM. Relationship between vertical ground reaction force and speed during walking, slow jogging, and running. Clin Biomech (Bristol, Avon). 1996; 111(5):253259.

17. Rosenbaum D, Hautmann S, Gold M, Claes L. Effects of walking speed on plantar pressure patterns and hindfoo angular motion. Gait \& Posture. 1994; 12(3):191 - 197.

18. Hennig EM, Staats A, Rosenbaum D. Plantar pressure distribution patterns of young school children in comparison to adults. Foot Ankle Int. 1994; 115(1):35-40.

19. Holm I, Tveter AT, Fredriksen PM, Vollestad N. A normative sample of gait and hopping on one leg parameters in children 7-12 years of age. Gait Posture. 2009; 129(2):317321.

20. Mickle KJ, Cliff DP, Munro BJ, Okely AD, Steele JR. Relationship between plantar pressures, physical activity and sedentariness among preschool children. J Sci Med Sport. 2011; 114(1):36-41.

\section{CORRESPONDÊNCIA}

Felipe P Carpes, Ph.D

Universidade Federal do Pampa;

BR 472 km 592

Cx postal 118 - CEP $97500-970$

Uruguaiana, RS, Brasil;

e-mail: carpes@unipampa.edu.br 